\title{
Extracting Vascular Networks under Physiological Constraints via Integer Programming
}

\author{
Markus Rempfler ${ }^{1}$, Matthias Schneider ${ }^{1}$, Giovanna D. Ielacqua ${ }^{2}$, \\ Xianghui Xiao ${ }^{3}$, Stuart R. Stock ${ }^{4}$, Jan Klohs ${ }^{2}$, Gábor Székely ${ }^{1}$, \\ Bjoern Andres ${ }^{5}$, and Bjoern H. Menze ${ }^{1,6}$ \\ ${ }^{1}$ Computer Vision Laboratory, ETH Zürich, Switzerland \\ 2 Institute for Biomedical Engineering, University and ETH Zürich, Switzerland \\ 3 Advanced Photon Source, Argonne National Laboratory, USA \\ ${ }^{4}$ Feinberg School of Medicine, Northwestern University, Chicago IL, USA \\ ${ }^{5}$ Max Planck Institute for Informatics, Saarbrücken, Germany \\ ${ }^{6}$ Institute for Advanced Study and Department of Computer Science, TU München, Germany
}

\begin{abstract}
We introduce an integer programming-based approach to vessel network extraction that enforces global physiological constraints on the vessel structure and learn this prior from a high-resolution reference network. The method accounts for both image evidence and geometric relationships between vessels by formulating and solving an integer programming problem. Starting from an over-connected network, it is pruning vessel stumps and spurious connections by evaluating bifurcation angle and connectivity of the graph. We utilize a high-resolution micro computed tomography $(\mu \mathrm{CT})$ dataset of a cerebrovascular corrosion cast to obtain a reference network, perform experiments on micro magnetic resonance angiography ( $\mu \mathrm{MRA})$ images of mouse brains and discuss properties of the networks obtained under different tracking and pruning approaches.
\end{abstract}

\section{Introduction}

Many diseases affect general properties of the cerebrovascular network, examples are arteriosclerosis and dilative vascular malformations changing vessel shape and diameter, but also Alzheimer's and related neuro-degenerative diseases that are suspected to affect the general vascularity and global network properties [1, 2]. While segmenting and tracing tubular structures is a longstanding field of interest in medical image computing [3-6], we approach here the wider - and somewhat neglected [7] - problem of extracting the full vascular network from image volumes under consideration of global geometric properties of the vascular structure.

Most vessel segmentation techniques rely on tubularity measures, e.g. [3], or other vessel enhancement filters, and further apply rule-based or learned decision algorithms to segment the vessels [5, 6, 8]. Usually, networks are extracted from the binary segmentations using morphological operators [9, 10], or by tracking vessels directly for which various methods have been proposed (as extensively reviewed in [5, 6]). Extracted structures often need to be further processed depending on the final application: $\mathrm{Lu}$ et al. [11], for example, proposed a supervised approach to vessel-structure parsing that included local geometrical features. Kaufhold et al. [12] discussed gap filling 
and pruning whereas Schneider et al. [13] recently proposed a generative approach to deal with imperfections in vascular connectivity of extracted networks. While segmentation algorithms are likely to enforce expected local vessel shape and geometry, only few approaches consider global physiological properties of the network when extracting the full network: Jiang et al. [14] incorporated assumptions about vessel diameters (Murray's hypothesis) in a global optimization problem for vascular trees. Here, Türetken et al. [15] introduced an integer programming approach that evaluates geometrical properties of curvilinear structures. Starting from an over-connected graph, they are pruning edges that do not fulfill desired structural relationships of neighbouring segments using a classifier that is trained from annotated 3-D networks (requiring manual annotation of both true positive and false positive connections).

In this paper, we enforce global geometrical constraints similar to Jiang et al. [14], but follow the inference approach by Türetken et al. [15]. We combine the global geometric prior with a local vessel segmentation algorithm [8] - i.e., we do not have to design individual classifiers for each geometrical constraint, but simply learn the global statistic of desired geometrical properties of the network from a high resolution dataset - and are able to trade local image evidence with global geometric properties as well as network connectivity. Identifying a new and more efficient scheme for the integer programming problem, we can now scale it to large datasets and illustrate its application for delineating vessels in $\mu \mathrm{MRA}$ images of the murine brain.

\section{Methods}

Segmentation Method. We transform image intensities into confidence maps by using the framework of Scheider et al. [8]: In this approach, multiscale steerable filter templates (SFT) are used as efficient directional filters, offering features that are invariant with respect to the local vessel direction. An oblique random forest (RF), which determines splits by solving a linear regression with elastic net penalty in each node, is used for a subsequent classification indicating the local presence of a vessel-like structure. The $\mathrm{RF}$ assigns each voxel $v$ in an image volume $I$ to a probability $p_{v} \in[0,1]$. We apply a threshold $\theta$ to the probability volume $P(I)$ and skeletonize the resulting binary volume using distance-ordered homotopic thinning (DOHT) [9], a method that iteratively removes voxels without altering the objects topology, to derive a network graph $G(\theta)$.

We obtain an over-connected network by generating multiple binary segmentations from $P(I)$ with different thresholds $\left\{\theta_{i}\right\}$, skeletonizing each of them by DOHT to $G\left(\theta_{i}\right)$ and superposing them into one network $G^{\text {over }}\left(\left\{\theta_{i}\right\}\right)$. The resulting network contains both segments with low confidence (contributed by graphs from low thresholds $\theta$ close to 0 ), but maintains the high spatial accuracy of a graph that is generated from conservative thresholds (i.e., with $\theta$ close to 1 ).

Vessel Network Regularization Using Integer Programming. Our regularization method starts with the over-connected vessel network graph $G^{\text {over }}=(V, E)$ with edges $E=\left\{e_{i}\right\}$ and image evidence $P(I)$. For every edge $e_{i} \in E$, we introduce a binary variable $x_{i}$ which indicates whether or not its corresponding edge is active (i.e. $x_{i}=1$ ). 
We then attempt to determine the optimal configuration of $\boldsymbol{x}$ by solving an integer programming problem. Our objective function $J(\boldsymbol{x})$ to be minimized is formulated as:

$$
J(\boldsymbol{x})=\alpha \sum_{x_{i} \in X} w_{i} x_{i}+\sum_{\substack{x_{i}, x_{j} \in X: \\
e_{i}, e_{j} \text { adjacent }}} w_{i j} x_{i} x_{j}+\sum_{\begin{array}{c}
x_{i}, x_{j}, x_{k} \in X: \\
e_{i}, e_{j}, e_{k} \text { adjacent }
\end{array}} w_{i j k} x_{i} x_{j} x_{k},
$$

where $w_{i}$ is the weight assigned to edge $e_{i}, w_{i j}$ the weight for adjacent pairs and $w_{i j k}$ for adjacent triplets. We identify the first sum - similar to [15] - as proportional to the negative $\log$-likelihood $-\log P(I, G \mid \boldsymbol{X}=\boldsymbol{x})$ of observing image $I$ and extracting graph $G$ given the underlying network represented by $\boldsymbol{x}$. In the same sense, we interpret the second and third term as geometric prior, $-\log P(\boldsymbol{X}=\boldsymbol{x} \mid \boldsymbol{\Theta})$, where $\boldsymbol{\Theta}$ is the parameter set of the model encoding physiologically realistic geometric relationships evaluating, for example, the plausibility of a bifurcation with the observed angle. The optimal solution $x^{*}$ is obtained by trading image evidence with expected geometric shape of a vessel network, a trade-off that can also be adjusted using parameter $\alpha>0$.

The optimization objective in (1) contains second and third order relations between variables. To deal with these, we introduce auxiliary binary variables $Y=\left\{y_{i j}\right\}$ and $Z=\left\{z_{i j k}\right\}$ to substitute second and third order terms in the objective. A set of linear constraints ties these auxiliary variables to the corresponding indicator variables $\left\{x_{i}\right\}$, leading to the following problem:

$$
J(\boldsymbol{x}, \boldsymbol{y}, \boldsymbol{z})=\alpha \sum_{x_{i} \in X} w_{i} x_{i}+\sum_{y_{i j} \in Y} w_{i j} y_{i j}+\sum_{z_{i j k} \in Z} w_{i j k} z_{i j k},
$$

s.t. $y_{i j} \leq x$

$y_{i j} \geq x_{i}+x_{j}-1$

$z_{i j k} \leq x$

$z_{i j k} \geq x_{i}+x_{j}+x_{k}-2$

$$
\begin{aligned}
\forall x \in\left\{x_{i}, x_{j}\right\}, \forall y_{i j} & \in Y, \\
\forall y_{i j} & \in Y, \\
\forall x \in\left\{x_{i}, x_{j}, x_{k}\right\}, \forall z_{i j k} & \in Z, \\
\forall z_{i j k} & \in Z .
\end{aligned}
$$

For the image evidence term in (1) we identify the weights $w_{i}$ with log likelihood ratios based on image evidence for edge $e_{i}$ corresponding to $x_{i}$ :

$$
w_{i}=-\log \frac{P\left(x_{i}=1 \mid I_{i}\right)}{P\left(x_{i}=0 \mid I_{i}\right)}=-\log \frac{p_{i}}{1-p_{i}},
$$

where we set $P\left(x_{i}=1 \mid I_{i}\right)=p_{i}$, the local confidence obtained by averaging along $e_{i}$ in the image evidence $P(I)$ obtained from the RF. For the auxiliary variables $Y$ and $Z$ of the geometric constraints - that we associate with the two states continue $\left(\Rightarrow y_{i j}=1\right)$ and $\operatorname{branch}\left(\Leftrightarrow z_{i j k}=1\right)$ - we identify weights $w_{i j}$ and $w_{i j k}$ that penalize or reward continuing or branching segments depending on their topological relationship. We set:

$$
\begin{aligned}
w_{i j} & =-\log \frac{P\left(y_{i j}=1 \mid \boldsymbol{\Theta}\right)}{P\left(y_{i j}=0 \mid \boldsymbol{\Theta}\right)}, \\
w_{i j k} & =-\log \frac{P\left(z_{i j k}=1 \mid \boldsymbol{\Theta}\right)}{P\left(z_{i j k}=0 \mid \boldsymbol{\Theta}\right)}-w_{i j}-w_{i k}-w_{j k},
\end{aligned}
$$

where we determine $P\left(y_{i j}=1 \mid \boldsymbol{\Theta}\right)=P\left(\right.$ continue $\left.\mid \gamma_{i j}, \boldsymbol{\Theta}\right)$ and $P\left(z_{i j k}=1 \mid \boldsymbol{\Theta}\right)=$ $P\left(\right.$ branch $\left.\mid \gamma_{i j k}, \Theta\right)$ according to the model $\Theta$ and the geometric features $\gamma$. The denominator may be modeled accordingly or be replaced by a tuning parameter. 
Fig. 1. Example of variables at a potential bifurcation. Segments, e.g. $x_{i}$, are drawn as solid black lines, while a pairwise variable $y_{i j}$ that represents $x_{i}$ continuing in $x_{j}$ is depicted by the dark grey overlay $\left(y_{i k}, y_{j k}\right.$ are omitted for clarity). $z_{i j k}$ corresponds to all three adjacent edges in the bifurcation (light grey). Furthermore, both deviation angles $\gamma_{\mathrm{d}}$ (blue) and the inner angle $\gamma_{\text {in }}$ (red) are shown. Radii are estimated perpendicular to the edge direction. In our experiments, we consider geometric features $\gamma_{i j k}=\left(\gamma_{\mathrm{in}}, \gamma_{\mathrm{d} 1}, \gamma_{\mathrm{d} 2}\right)_{i j k}$ for bifurcations and $\boldsymbol{\gamma}_{i j}=\left(\gamma_{\mathrm{d}}\right)_{i j}$ for continuing segments, while radius estimates are used to determine the main trunk.

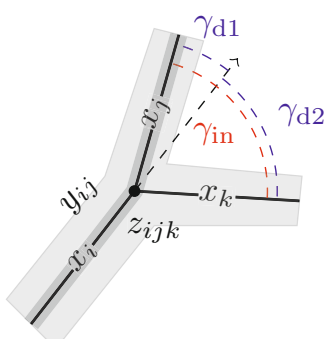

Solving the Integer Programming Problem for Large Datasets. The described integer programming problem of (2) with the associated constraints can be approximated by a branch and cut algorithm implemented in libraries such as [16]. However, the optimal solution to this problem is not necessarily a plausible vessel network yet and connectivity constraints might be required. Since both auxiliary variables and constraints grow quickly in number for larger graphs, such as to be expected from a complete brain scan, we propose to employ a lazy constraint generation scheme, i.e. only add necessary constraints in an iterative manner, together with the following approach:

1. Given the variable set $X$ of (1), we define a graph $A=\left(V_{A}, E_{A}\right)$ with a vertex $v_{i} \in V_{A}$ for every $x_{i} \in X$.

2. An edge $e_{i j}$ is added to $E_{A}$ if and only if there exists a constraint that contains both variables $x_{i}$ and $x_{j}$.

3. Determine the connected components in $A$. Vertices of each connected component represent a sub-problem that can be solved independently - of course with their according constraints.

Whenever a constraint or variable is added, we adjust this structure dynamically. Then only sub-problems, i.e. connected components, that are affected by the change need to be re-solved (e.g. by a standard solver) speeding up the performance over approaches that solve the complete problem in every run.

\section{Experiments}

Image Data and Preprocessing. We use four 3-D $\mu \mathrm{MRA}$ images of the murine brain of size $248 \mathrm{px} \times 248 \mathrm{px} \times 109 \mathrm{px}$ with an isotropic voxel spacing of $60 \mu \mathrm{m}$, and a corrosion cast $\mu \mathrm{CT}$ of the cerebral vessel network of a mouse with a volume of $1024 \mathrm{px} \times$ $1024 \mathrm{px} \times 1857 \mathrm{px}$ voxels, with a spacing of $5.8 \mu \mathrm{m}$.

We apply the segmentation framework of Schneider et al. [8], adjusting filter template order and scales as well as random forest parameters in a leave-one-out crossvalidation using manually annotated ground truth labels. Probability maps $P(I)$ are binarized for different thresholds $\theta$ and transformed into network graphs using DOHT as discussed above. We segment and track vessels in both $\mu \mathrm{MRA}$ and the $\mu \mathrm{CT}$ volume. 

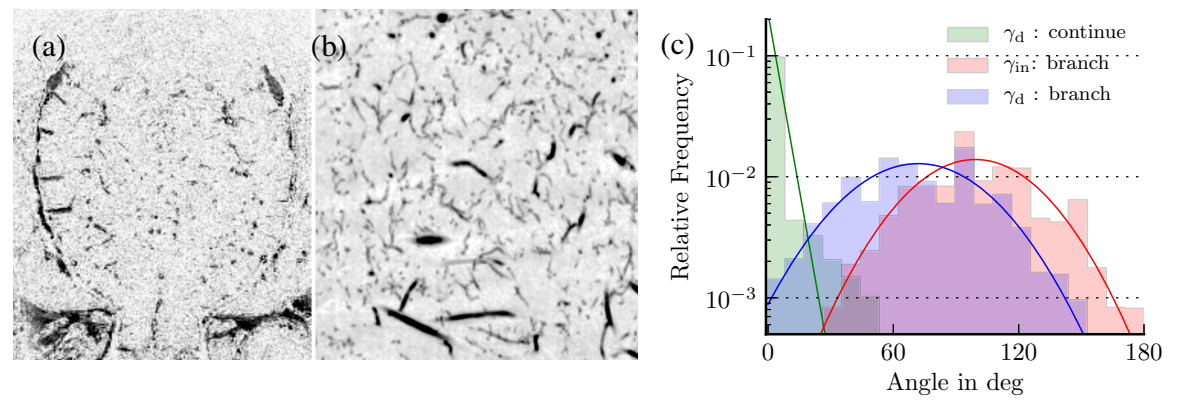

Fig. 2. (a) Example of a whole-brain $\mu \mathrm{MRA}$ dataset (gray-scale inverted), (b) corrosion cast $\mu \mathrm{CT}$ subregion of $256 \mathrm{px} \times 256 \mathrm{px}$, (c) angle histograms computed on the extracted network of the corrosion cast dataset with inferred distributions (solid). Shown are deviation angles of continuing segments (green), deviation (blue) and inner (red) bifurcation angles (see Fig. 11.

Training: Learning the Geometric Model from the High Resolution Network. We use the geometrical prior to support bifurcations in our over-connected graph that are valid with respect to their diameter and relative angle, and remove those that are not. To this end, we learn the relative frequencies of radii and deviation angles of vessel segments from the high resolution $\mu \mathrm{CT}$ (see Fig. 2 k for angles). We find $p(\gamma \mid$ continue, $\boldsymbol{\Theta}$ ) to be well represented by an exponential distribution (where $\gamma$ is the deviation angle between two continuing vessels), and $p(\gamma \mid$ branch, $\boldsymbol{\Theta})$ to be well represented by a Gaussian (where $\gamma$ are the three angles of a bifurcation) while $\Theta$ is the joint set of parameters of the two distribution models. Radius estimates are utilized to determine which vessel is the main trunk in a bifurcation and the reference for the angles considered here. The model is utilized to determine weights $w_{i j}$ and $w_{i j k}$ as in (8) and (9) with the help of Bayes' formula, and assuming a uniform prior on $P$ (continue) and $P$ (branch). For the weights' denominator, we use a uniform distribution for pairs (i.e. $P(\gamma \mid \neg$ continue $) \sim \mathcal{U}(\gamma)$ ) and model $P(\gamma \mid \neg$ branch $)$, for bifurcations, by the probability of any involved pair being continuing. Parameters $\Theta$ are fitted to the distributions observed in the $\mu \mathrm{CT}$ using the maximum likelihood estimate.

Experiment: Trading Image Evidence with Geometrical Prior. In Fig. 3, the behaviour of our regularization method with varying weight parameter $\alpha$ is shown. For large values of $\alpha$, the image evidence is emphasised (the network is still connected), while choosing a small value of $\alpha$ puts more emphasis on geometric weights. Although varying $\alpha$ has no large impact in our case, it may be advisable for other problems to determine the most suitable $\alpha$ depending on the previously applied processing.

Experiment: Applying the Network Model to Low Resolution $\mu$ MRA Data. We generate an over-connected graph for each of the $\mu \mathrm{MRA}$ test sets using the approach described in Sect. 2 with multiple thresholds $\theta_{i} \in\{0.2,0.5,0.9\}$. After removing unphysiological nodes and edges, we compare the regularized network to unregularized networks obtained from individual thresholds $\theta=0.2,0.5$ and 0.9 . 

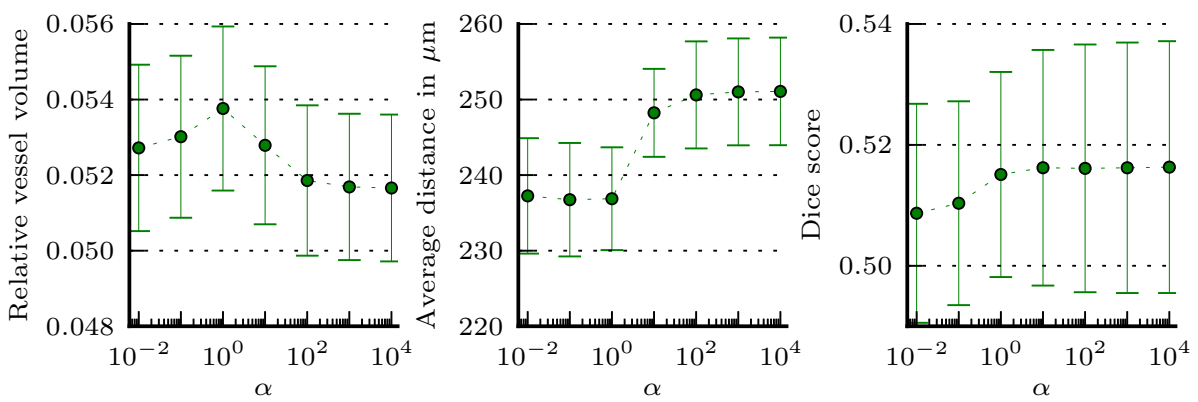

Fig. 3. Sensitivity study on the weight parameter $\alpha$ performed on two $\mu \mathrm{MRA}$ datasets. The parameter $\alpha$ has a limited impact with respect to the depicted scores. Averages are within $5.16 \%$ and $5.37 \%$ for relative vessel volume, $236 \mu \mathrm{m}$ and $251 \mu \mathrm{m}$ average distance and $50.8 \%$ and $51.6 \%$ Dice.
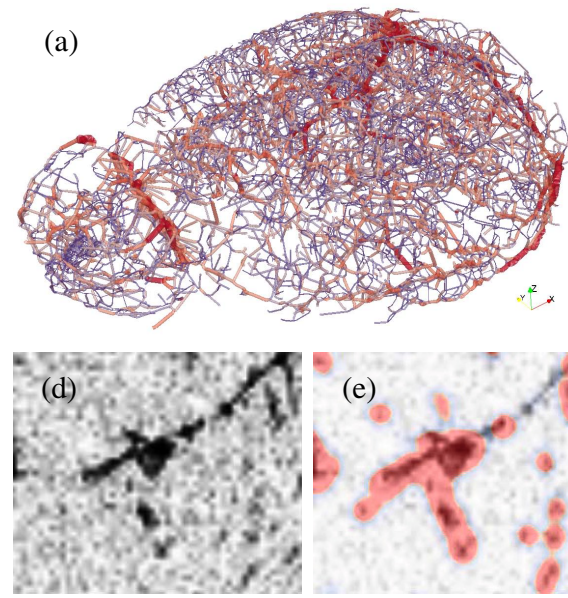

(e)

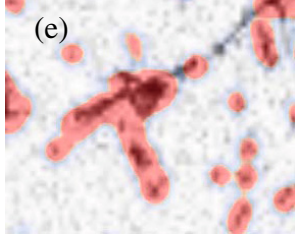

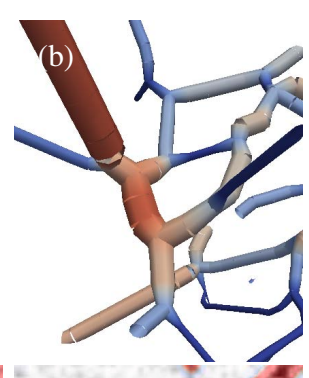

(f)

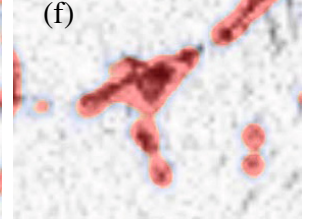

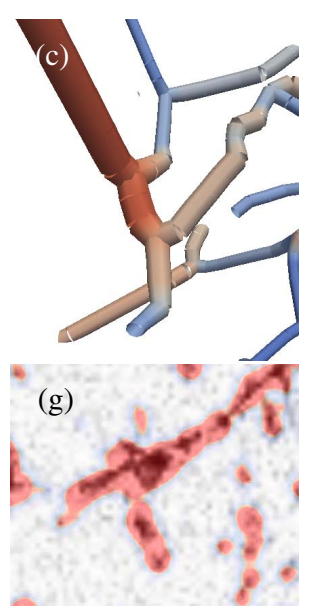

Fig. 4. Visualisation of the results. (a) Rendered vascular network extracted with our method. (b) Detail view before and (c) after regularization. Colours change with vessel diameter. (d) Raw image. (e) Rasterized DOHT network $\theta=0.5$ and (f) with $\theta=0.9$. (g) Postprocessed network with our method. Note that for the rasterization, a simple tube model is used and therefore, not a perfect voxel-grained segmentation is to be expected but rather a qualitative visualization that indicates whether or not a structure is present in the network model (shown as red overlay).

Figure 4a shows a regularized network, while Fig. 4b-c provides close-up views before and after regularization, respectively. A number of spurious sprouts and loops are visible that are removed during regularization. Details in Fig. $4 \mathrm{~d}-\mathrm{g}$ show differences between the two thresholded and a regularized network. We note that both thresholded networks (Fig. 4 e-f) lose the connection of the large branch (center to top-right), while it is retained in the regularized network (Fig. $4 \mathrm{~g}$ ).

Figure 5 reports quantitative measures of global network properties, such as total vessel volume, average distance to the next vessel (i.e. extravascular distance) and Dice 

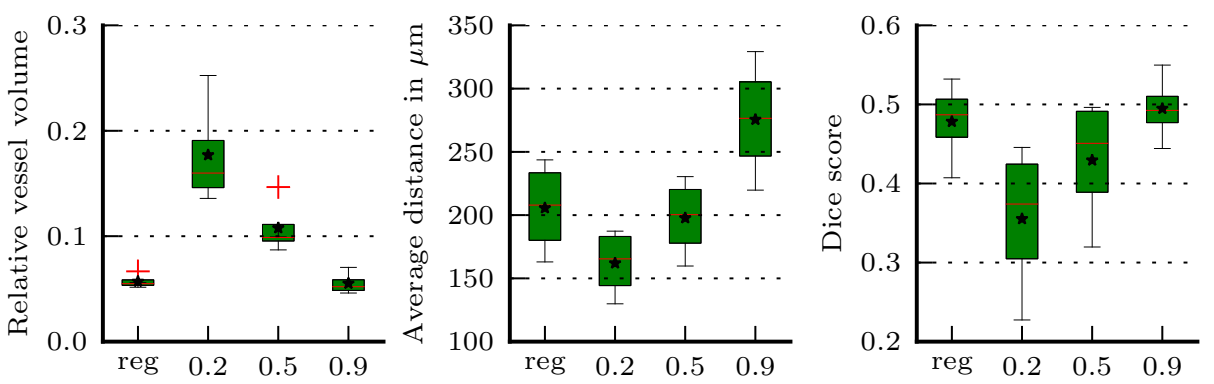

Fig. 5. Comparison of the regularized network (reg) with unregularized networks obtained at thresholds $\theta=0.2,0.5$ and 0.9 . Boxplots (median in red, mean as $\star$ ) depict the statistics on all four $\mu$ MRA datasets. The Dice score has been computed by rasterizing the network using a simple tube model (negatively affecting absolute Dice values for all approaches to the same degree) and comparing it to voxel-based segmentation labels. For this experiment, $\alpha=1$ was chosen. Our regularization always groups with the more favourable of the alternatives, i.e. it has a small vessel volume, a moderate average distance and yet an acceptable high Dice score.

score when rasterized (using a tubular model) and compared with voxel-based segmentation labels. We find the regularized network to always group with the more favourable of the alternative, which is a low vessel volume, a moderate, i.e. neither too small nor too large, extravascular distance and a high Dice score, while each of the alternative approaches provides poor results in at least one of these scores.

Experiment: Scaling to Large Datasets. Comparing the processing time of the proposed solver (Sect. 2]) and a standard method (IBM ILOG CPLEX V12.51 [16]), we find that our approach of handling subproblems during the optimization leads to an improvement: average run times (on a quadcore CPU, 8 GB RAM, same set-up as in the previous experiment) for 10 repeated runs on three test datasets were 25.0, 9.5, 34.9 [min] for our approach, while the standard solver required $57.8 \% \pm 0.39 \%, 56.1 \% \pm 0.22 \%$ and $20.2 \% \pm 1.83 \%$ longer run times, respectively. Note that absolute run times may vary widely depending on data and parametrization due to the dynamic processing scheme.

\section{Conclusions}

We have introduced a regularization method for vessel networks and presented experiments on $\mu \mathrm{MRA}$ images where we learned the physiological-geometric prior from a high-resolution corrosion cast $\mu \mathrm{CT}$ of a murine cerebrovascular network. Our method can be applied as post-processing step to existing vessel segmentation pipelines in order to incorporate physiological knowledge for improved network extraction. It is easily extendable to other physiological prior, for example considering vessel shape, length, network structure or prior learning as in [11], and it can also be combined with other geometrical or physiological re- and over-connection schemes such as discussed in [12, 13].

Acknowledgements. This research was supported by the Technische Universität München - Institute for Advanced Study (funded by the German Excellence Initiative and the European Union Seventh Framework Programme under grant agreement 
n 291763, the Marie Curie COFUND program of the the European Union), by grants from the EMDO foundation, Swiss National Science Foundation grant 136822, and the Swiss National Center of Competence in Research on Computer Aided and Image Guided Medical Interventions (NCCR Co-Me) supported by the Swiss National Science Foundation. Use of the Advanced Photon Source was supported by the US Department of Energy, Office of Science, Office of Basic Energy Sciences, under Contract No. DE-AC02-06CH11357.

\section{References}

1. Klohs, J., et al.: Contrast-enhanced magnetic resonance microangiography reveals remodeling of the cerebral microvasculature in transgenic arca $\beta$ mice. J. Neurosc. 32, 1705-1713 (2012)

2. Hunter, J.M., et al.: Morphological and pathological evolution of the brain microcirculation in aging and Alzheimer's disease. PLoS ONE 7, e36893 (2012)

3. Frangi, A.F., Niessen, W.J., Vincken, K.L., Viergever, M.A.: Multiscale vessel enhancement filtering. In: Wells, W.M., Colchester, A.C.F., Delp, S.L. (eds.) MICCAI 1998. LNCS, vol. 1496, pp. 130-137. Springer, Heidelberg (1998)

4. Aylward, S., Bullitt, E.: Initialization, noise, singularities, and scale in height ridge traversal for tubular object centerline extraction. IEEE TMI 21, 61-75 (2002)

5. Kirbas, C., Quek, F.: A review of vessel extraction techniques and algorithms. ACM Comput. Surv. 36, 81-121 (2004)

6. Lesage, D., et al.: A review of 3D vessel lumen segmentation techniques: Models, features and extraction schemes. Medical Image Analysis 13, 819-845 (2009)

7. Jiang, Y., et al.: Vascular tree reconstruction by minimizing a physiological functional cost. In: Proc. CVPR Workshops, pp. 178-185 (2010)

8. Schneider, M., Hirsch, S., Székely, G., Weber, B., Menze, B.H.: Oblique random forests for 3-D vessel detection using steerable filters and orthogonal subspace filtering. In: Menze, B.H., Langs, G., Lu, L., Montillo, A., Tu, Z., Criminisi, A. (eds.) MCV 2012. LNCS, vol. 7766, pp. 142-154. Springer, Heidelberg (2013)

9. Pudney, C.: Distance-ordered homotopic thinning: A skeletonization algorithm for 3D digital images. CVIU 72, 404-413 (1998)

10. Lee, T., et al.: Building skeleton models via 3-D medial surface axis thinning algorithms. Graphical Models and Image Processing 56, 462-478 (1994)

11. Lu, L., et al.: Hierarchical learning for tubular structure parsing in medical imaging: A study on coronary arteries using 3D CT angiography. In: Proc. ICCV, pp. 2021-2028 (2009)

12. Kaufhold, J.P., et al.: Vectorization of optically sectioned brain microvasculature: Learning aids completion of vascular graphs by connecting gaps and deleting open-ended segments. Medical Image Analysis 16, 1241-1258 (2012)

13. Schneider, M., Hirsch, S., Weber, B., Székely, G., Menze, B.H.: TGIF: Topological gap infill for vascular networks - A generative physiological modeling approach. In: Golland, P., Hata, N., Barillot, C., Hornegger, J., Howe, R. (eds.) MICCAI 2014. LNCS, vol. 8674, pp. 89-96. Springer, Berlin (2014)

14. Jiang, Y., Zhuang, Z.W., Sinusas, A.J., Staib, L.H., Papademetris, X.: Vessel connectivity using murray's hypothesis. In: Fichtinger, G., Martel, A., Peters, T. (eds.) MICCAI 2011, Part III. LNCS, vol. 6893, pp. 528-536. Springer, Heidelberg (2011)

15. Türetken, E., et al.: Reconstructing loopy curvilinear structures using integer programming. In: Proc. CVPR, pp. 1822-1829 (2013)

16. IBM ILOG CPLEX Optimizer (2014), http: / / www . ibm. com/software/ integration/optimization/cplex-optimizer/ 